\title{
The situation, problems and measures of Chinese herbal medicine about producing mode in Shandong Province
}

\author{
Xin $\mathrm{Su}^{1, \mathrm{a}}$, Xiaoting $\mathrm{Gao}^{2, \mathrm{~b}}$, Renzhu $\mathrm{Yu}^{3, \mathrm{c}}$ \\ ${ }^{1}$ School of Business Administration Shandong University of Finance and Economic \\ No.7366,East Erhuan Road,Jinan,250014 China \\ ${ }^{2}$ School of Business Administration Shandong University of Finance and Economic \\ No.7366, East Erhuan Road,Jinan,250014 China \\ ${ }^{3}$ School of Business Administration Shandong University of Finance and Economic \\ No.7366,East Erhuan Road,Jinan,250014 China

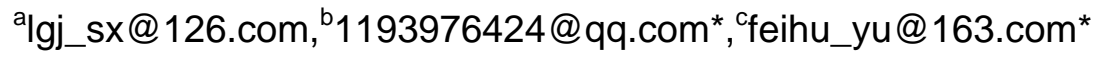 \\ (*means corresponding author)
}

\begin{abstract}
Keywords: the mode of Chinese herbal medicine; the producing mode about the whole family; the producing mode which more people cooperate
\end{abstract}

\begin{abstract}
Chinese herbal medicine is not only a traditional treasure, but also promotes the development. But the producing mode is bad for the development of Chinese herbal medicine.Now, the article chooses rich resources of Shandong province to conduct research, which to understand the producing mode about Chinese herbal medicine-the producing mode about the whole family and the producing mode which more people cooperate. Through analysing the situation about the mode of Chinese herbal medicine,we can find out the existing problems and put forward corresponding recommendations.
\end{abstract}

\section{Introduction}

Chinese herbal medicine is not only the important culture, but also is the basic of traditional Chinese medicine. Compared with western medicine, Chinese herbal medicine is more good for our health and more conducive to rehabilitation of patients. So it becomes an important choice for the sick people and provides a powerful impetus to developming the Chinese herbal medicine. Through conducting research about the rich resources of Shandong province, we can find that the model of the Chinese herbal medicine is bad for its development. Now, more people can choose the producing mode about the whole family and they are scattered everywhere. So it is bad for expanding planting area and standarding operating procedure.

Through the analysis, we can analysis the situation of the producing model and put forward existing problems.it is important for promoting the reform of the producing mode and developmenting the Chinese herbal medicine.

Firstly, through conducting the research, we can find existing problems. More people choose the producing mode about the whole family in Shandong Province. They do not have advanced technology and are difficult to expanding planting area.

Secondly, through the analysis of the problem, we can put forward countermeasures and suggestions about the producing model of the Chinese herbal medicine. It is good for promoting the sustainable development of Chinese herbal medicine.

In a word, through the study of the producing mode of the Chinese herbal medicine, it is of great practical significance for promoting the long-term and stable development of Chinese herbal medicine. 


\section{Analysing the current situation of the producing mode about Chinese herbal in Shandong} Province

After years, there are two main producing modes about Chinese herbal medicine in Shandong Province: Chinese herbal medicine-the producing mode about the whole family and the producing mode which more people cooperate (vertical and horizontal).

\subsection{The producing mode which the whole family join}

The basic way of the producing mode about the Chinese herbal medicine in Shandong province is the family as a unit to product, and most farmers do not use the entire contracted land to cultivate. With the improvement of mechanization,the cultivation and transportation, fertilizer and harvesting about the Chinese herbal medicine are mostly automated. There is not need other labor force, mainly relying on family members. At the same time,choosing the producing mode about the Chinese herbal medicine has also been affected by technology.

\subsection{The producing mode which more people cooperate}

Since the beginning of the new century, the producing model about the Chinese herbal medicine in Shandong province has also undergone tremendous changes.The producing model is affected by the producing model about vegetables and other producing organizations. The producing model about the Chinese herbal medicine in Shandong Province has two ways, one is the horizontal organization, mainly refers to the organization between producers and operators, such as the Technology Association about the Chinese Herbal Medicine; two is the vertical organization,namely the organization between enterprises.

\subsubsection{The horizontal organization about the Chinese herbal medicine}

At present,the majority of producer in Shandong province are professional associations, and all are professional associations based on technical services. Farmers voluntarily join in the association,and provide technical organization only. Therefore,the interests of the organizations are relatively loose. The technology association about the Chinese Herbal Medicine in Shandong Province takes the lead in the establishment of agricultural technology.

\subsubsection{The Vertical organization about the Chinese herbal medicine}

The vertical organization about the Chinese herbal medicine refers to producers and marketers. In order to improving producing efficiency and enhancing competitive advantage,they establish stable cooperative relations between supply and demand by contracting relationship in the production, marketing and using raw materials.There are mainly forward and backward organization.

3Problems existing in the producing model about the Chinese herbal medicine in Shandong province

\subsection{Chinese herbal medicine-the producing mode about the whole family}

\subsubsection{Chinese herbal medicine-the producing mode about the whole family lacks obvious interests}

Although the Chinese herbal medicine-the producing mode about the whole family has some advantages, such as stable production, less human input.Compared with the collective farming inputs,only inherent planting area and machinery to help ridge transportation, this does not conform to the characteristics of Chinese herbal medicine.At the same time, almost all the inputs are human factors and they already belong to a typical labor-intensive industry. The effect of the Chinese herbal medicine about the supply and demand information,Chinese herbal medicine-the producing 
mode about the whole family is generally small.

\subsubsection{The constraints of land resources}

The Chinese herbal medicine mainly rely on land resources, especially famers need more land. But the impact of land resources about the Chinese herbal medicine is mainly manifested in two aspects: one is that with the continuous cropping, rotation of crops is restricted; two is the land and water pollution.

\subsection{The producing mode which more people cooperate-horizontal organization}

\subsubsection{Farmers lack enthusiasm to enter the organization}

Most farmers do not agree with organization, but large farmers are interested in entering organization. The survey found that farmers who are merged into the organization do not have a strong desire to cooperate and they do not have enthusiasm.

\subsubsection{Orgnization lacks funds which restrict work to play}

The planting scale is generally small in Shandong province. Some farmers only see the Chinese herbal medicine as "sideline", and the land is difficult to concentrate. This situation do not expand the planting scale. Famers do not pay for funds by planting small scale. The newly formed organization can not finance through bank loans. Because they lack a good credit.

\subsection{The producing mode which more people cooperate-vertical organization}

\subsubsection{The vertical organization has a narrow range of services and is difficult to meet the needs of member}

The vertical organization about the Chinese herbal medicine can not meet the needs of members, and the service is relatively narrow. The vertical organization about the Chinese herbal medicine has played a certain role in introducing and popularizing new varieties, but it can not obtain high benefit. Because it can not meet the needs of members.

\subsubsection{The vertical organization lacks talent and funds}

The members of the vertical organization are the main source of funding. The organization does not provide good services for members, so cooperative members are not willing to provide the corresponding activity funds and reduce enthusiasm. The situation indirectly lead to the cooperative difficulties. The vertical organization are hampered by insufficient funds.

\section{The countermeasures and suggestions about the producing Model of the Chinese herbal medicine in Shandong Province}

\subsection{Expanding planting area}

At present, the main factors of restricting the development of Chinese herbal medicine in Shandong province is the small planting area and famers are scattered everywhere. This situation is not conducive to using new varieties and promoting the development of new technology. Indirectly, the producing model of the Chinese herbal medicine can waste resources, which is not conducive to the sustainable development of Chinese herbal medicine. Firstly, farmers need speed up the transfer of the right of rural land-use and actively establish rural land market.

\subsection{To improving the organizational level and the competitiveness about the Chinese herbal medicine}

At present, the famers have low level of organization in Shandong province. The government 
should actively guide the local farmers to join cooperatives. It is not only promote the development of cooperatives, but also improve the degree of organization about the Chinese herbal medicine. This is conducive to solve the problem of decentralized management and Chinese herbal medicine production over dispersion, Chinese herbal guide the producers care and protect the common interests.

\subsection{To strengthen publicity and education about the cooperative organizations}

In the economy market where exist in competition and organization, most people do not realize the importance of organization. Because they want to gain profits by competing with others. Therefore, in the process of promoting the further development of Chinese herbal medicine, the most important thing is mobilize the majority of farmers to actively cooperate with others. At the same time, the government should strengthen publicity and education.

\subsection{To promote the expansion of Chinese herbal medicine producers and industrial market players}

The cooperatives has an important problem is the stability of Chinese herbal medicine. In order to solving this problem, the most important way is promote the large-scale expansion of Chinese herbal medicine producers in Shandong province. The government should strengthen land circulation and guarantee famers gain land by signing a contract.

\section{Acknowledgement}

This work is partially supported by1. the National Philosophy and Social Science Foundation as an important program, the Assurance Mechanism of Agricultural Product Quality and Safety based on Multi-agent Coordination(No. 15AGL014). It is also partially supported by 2.the Important Research and Development Program of Shandong Province in 2016, Key Issues and System Implementation of Early Warning for Agricultural Product Quality Safety under the Trend of Intelligent Agriculture(No. 2016GSF120013). 3.the Technology System Innovation Plan of Chinese Herbal Medicine Industry of Shandong Province on the 13th Five-Year Plan, The authors would like to acknowledge the referees for their valuable comments and suggestions which helped to improve the presentation of this paper.

\section{References}

[1] Liang Xue,Yongkun Yang.Practice and Countermeasures of family farm's development[J].Agricultural economic problems.2015(02) 4-8

[2] Xin Su,Renzhu Yu,Xiaoyu Zhang.Research on the design of credit mechanism of agricultural products in China*[J].Macroeconomic Research.2014(04) 10-26

[3] Lunlai Wan,Jiaojiao Ma,Hugen Zhu,Organizational model of agricultural management and technical efficiency of leading enterprises in China- Empirical Evidence from leading enterprises of agricultural comprehensive development in Anhui[J].Chinese rural economy,2010(10) 27-35

[4] Puqing Wang,Deyi Zhou,Zhixuan Lv.The model of agricultural product and food safety about supply chain*[J].Agricultural economic problems,2009(03) 8-12

[5] Huifeng Zhao,Tong Li.Analysis of the mode of agricultural industrialization in foreign countries[J].Agricultural economic problems,2002(02) 60-63

[6] Minglin Zhang,Yaobin Liu.Efficiency comparison of the model of agricultural industrialization: a cooperative game[J].Statistics and decision making,2007(21) 52-54

[7] Ruqiao Lei,Jixiang Chen,Qin Liu.Modular the model and its efficiency comparison[J].Chinese industrial economy,2004(10) 83-90 